\title{
Revista Brasileira de Educação do Campo
}

\section{[PT] Nominata de Pareceristas e Avaliadores ad hoc da Revista Brasileira de Educação do Campo em 2020}

\section{[EN] List of reviewers and referees of the Brazilian Journal of Rural Education}

\begin{tabular}{|c|c|}
\hline NOME / NAME & INSTITUIÇÃO / INSTITUTION \\
\hline ADLANDIA DO NASCIMENTO DIAS & UNIVERSIDADE DE PERNAMBUCO - UPE \\
\hline ALBERTO DIAS VALADÃO & UNIVERSIDADE FEDERAL DE RONDÔNIA - UNIR \\
\hline ALEXANDRE LEITE DOS SANTOS SILVA & UNIVERSIDADE FEDERAL DO PIAUI - UFPI \\
\hline ALTAIR ALBERTO FÁVERO & UNIVERSIDADE DE PASSO FUNDO - UPF \\
\hline ANA CRISTINA DAXENBERGER & UNIVERSIDADE FEDERAL DA PARAÍBA - UFPB \\
\hline ANA ELISA SPAOLONZI & UNIVERSIDADE ESTADUAL DE CAMPINAS - UNICAMP \\
\hline ANDREIA NUNES MILITAO & $\begin{array}{l}\text { UNIVERSIDADE ESTADUAL DE MATO GROSSO DO SUL - } \\
\text { UEMS }\end{array}$ \\
\hline ALCEU ZOIA & UNIVERSIDADE DO ESTADO DE MATO GROSSO - UNEMAT \\
\hline ALINE GUTERRES FERREIRA & $\begin{array}{l}\text { UNIVERSIDADE FEDERAL DO RIO GRANDE DO SUL - } \\
\text { UFRGS }\end{array}$ \\
\hline AMANDA MOTTA CASTRO & UNIVERSIDADE FEDERAL DO RIO GRANDE - FURG \\
\hline ANA PAULA CAETANO & PESQUISADORA \\
\hline ANA PAULA LIMA AZEVEDO & UNIVERSIDADE FEDERAL DO CEARÁ - UFC \\
\hline ARLINDO LINS MELO JUNIOR & UNIVERSIDADE FEDERAL DE SÃO CARLOS - UFSCAR \\
\hline ARLETE RAMOS DOS SANTOS & $\begin{array}{l}\text { UNIVERSIDADE ESTADUAL DO SUDOESTE DA BAHIA - } \\
\text { UESB }\end{array}$ \\
\hline BEATRICE ICHERIA & UNIVERSIDADE DE PRETORIA, AFRICA DO SUL \\
\hline BRUNO BARONNET & UNIVERSIDAD VERACRUZANA, VERACRUZ, MÉXICO \\
\hline CARLOS GAIA & $\begin{array}{l}\text { UNIVERSIDADE FEDERAL DO SUL E SUDOESTE DO PARÁ - } \\
\text { UNIFESSPA }\end{array}$ \\
\hline CARLOS LAURA & UNIVERSIDADE FEDERAL DE UBERLANDIA - UFU \\
\hline CARLOS ROBERTO MASSAO HAYASHI & UNIVERSIDADE \\
\hline CHARLES ALBERTO DE SOUZA ALVES & INSTITUTO FEDERAL DO AMAPÁ - IFPA \\
\hline CHERLEI MARCIA COAN & UNIVERSIDADE FEDERAL DA FRONTEIRA SUL - UFFS \\
\hline CÁSSIA FERREIRA MIRANDA & UNIVERSIDADE FEDERAL DO TOCANTINS - UFT \\
\hline CASSIA HACK & UNIVERSIDADE FEDERAL DO AMAPÁ - UFAP \\
\hline CASSYO LIMA SANTOS & UNIVERSIDADE FEDERAL DO TOCANTINS - UFT \\
\hline CINTIA MORALLES CAMILLO & UNIVERSIDADE FEDERAL DE SANTA MARIA - UFSM \\
\hline CLAUDEMIR DE QUADROS & UNIVERSIDADE FEDERAL DE SANTA MARIA - UFSM \\
\hline CRISTIENE ADRIANA DA SILVA CARVALHO & UNIVERSIDADE FEDERAL DE MINAS GERAIS - UFMG \\
\hline CRISTIANE TALITA GROMANN & UNIVERSIDADE FEDERAL DE RONDONIA - UNIR \\
\hline CHRISTIANO CORREA TEIXEIRA & $\begin{array}{l}\text { UNIVERSIDADE FEDERAL DO RIO GRANDE DO SUL - } \\
\text { UFRGS }\end{array}$ \\
\hline CLEIDE CARVALHO DE MATOS & UNIVERSIDADE FEDERAL DO PARÁ - UFPA \\
\hline CLAUDIO RODRIGUES SILVA & UNIVERSIDADE ESTADUAL PAULISTA - UNESP \\
\hline DEYLA PAULA DE OLIVEIRA & UNIVERSIDADE FEDERAL DO TOCANTINS - UFT \\
\hline DENISE DE OLIVEIRA ALVES & UNIVERSIDADE FEDERAL DE GOIAS - UFG \\
\hline DANIELA FAGUNDES PORTELA & UNIVERSIDADE DE SÃO PAULO - USP \\
\hline DANIELA FARIA FLORENCIO & $\begin{array}{l}\text { UNIVERSIDADE FEDERAL RURAL DO SEMI ÁRIDO - } \\
\text { UFERSA }\end{array}$ \\
\hline DUŠAN STAMENKOVIĆ & UNIVERSITY OF NIŠ, SÉRVIA \\
\hline DORACY DIAS DE AGUIAR CARVALHO & UNIVERSIDADE FEDERAL DO TOCANTINS - UFT \\
\hline EDSON CAETANO & UNIVERSIDADE FEDERAL DE MATO GROSSO - UFMT \\
\hline ELAINE SILVIA DUTRA & UNIVERSIDADE DO ESTADO DE MATO GROSSO - UNEMAT \\
\hline
\end{tabular}




\begin{tabular}{|c|c|}
\hline JOSÉ CARLOS FREIRE & UNIVERSIDADE FEDERAL DO TOCANTINS - UFT \\
\hline ELAINE MASCHIO & UNIVERSIDADE FEDERAL DO PARANÁ - UFPR \\
\hline EMERSON BASTOS LOMASSO & $\begin{array}{l}\text { PONTIFÍCIA UNIVERSIDADE CATÓLICA DE SÃO PAULO - } \\
\text { PUC/SP }\end{array}$ \\
\hline ELIANA POJO TOUTONGE & UNIVERSIDADE FEDERAL DO PARÁ - UFPA \\
\hline ELIEZER NUNES SILVA & INSTITUTO FEDERAL DE RORAIMA - IFRR \\
\hline ELOY ALVES FILHO & UNIVERSIDADE DE UBERABA - UNIUBE \\
\hline FABIANA FÁTIMA CHEROBIN & UNIVERSIDADE FEDERAL DE SANTA CATARINA - UFSC \\
\hline ELIUD TORRES & $\begin{array}{l}\text { UNIVERSIDAD AUTÓNOMA METROPOLITANA - UNIDAD } \\
\text { XOCHIMILCO, MÉXICO }\end{array}$ \\
\hline FABRICIA DA SILVA MACHADO & INSTITUO FEDERAL DO MARANHÃO - IFMA \\
\hline FABRICIO NILO LIMA DA SILVA & INSTITUTO FEDERAL DO PARÁ - IFPA \\
\hline FAGNER FREIRES DE SOUSA & INSTITUTO FEDERAL DO PARÁ - IFPA \\
\hline FLÁVIO PEREIRA DE OLIVEIRA & $\begin{array}{l}\text { UNIVERSIDADE FEDERAL RURAL DE PERNAMBUCO - } \\
\text { UFRPE }\end{array}$ \\
\hline FERNANDA MONTEIRO BARRETO CAMARGO & UNIVERSIDADE FEDERAL DO ESPIRITO SANTO - UFES \\
\hline FERNANDO JOSÉ MARTINS & $\begin{array}{l}\text { UNIVERSIDADE ESTADUAL DO OESTE DO PARANÁ - } \\
\text { UNIOESTE }\end{array}$ \\
\hline FLÁVIO PEREIRA DE OLIVEIRA & UNIVERSIDADE FEDERAL DA PARAÍBA - UFPB \\
\hline FRANCISCA RODRIGUES LOPES & UNIVERSIDADE FEDERAL DO TOCANTINS - UFT \\
\hline FRANCINEI BENTES TAVARES & UNIVERSIDADE FEDERAL DO PARÁ - UFPA \\
\hline GLAUCIA SOUSA MORENO & $\begin{array}{l}\text { UNIVERSIDADE FEDERAL DO SUL E SUDESTE DO PARÁ - } \\
\text { UNIFESSPA }\end{array}$ \\
\hline GRACIELE KRAEMER & $\begin{array}{l}\text { UNIVERSIDADE FEDERAL DO RIO GRANDE DO SUL - } \\
\text { UFRGS }\end{array}$ \\
\hline GREICE DE SOUSA & PESQUISADORA INDEPENDENTE \\
\hline HELDER PINTO & $\begin{array}{l}\text { UNIVERSIDADE FEDERAL DOS VALES DO } \\
\text { JEQUITINHONHA E MUCURI }\end{array}$ \\
\hline HELENA MARIA BELING & UNIVERSIDADE FEDERAL DE SANTA MARIA \\
\hline IDEMAR VIZOLLI & UNIVERSIDADE FEDERAL DO TOCANTINS - UFT \\
\hline IDALENA OLIVEIRA CHAVES & UNIVERSIDADE FEDERAL DE VIÇOSA - UFV \\
\hline IDALINA SOUZA BORGHI & $\begin{array}{l}\text { UNIVERSIDADE FEDERAL DO RECÔNCAVO DA BAHIA - } \\
\text { UFRB }\end{array}$ \\
\hline ILDETE FALCÃO & UNIVERSIDADE FEDERAL DO PARÁ - UFPA \\
\hline IRANILDO SILVA OLIVEIRA & CENTRO EDUCACIONAL MARANHENSE \\
\hline ISABEL MARTINS & UNIVERSIDADE FEDERAL DE MINAS GERAIS - UFMG \\
\hline ILDETE DA SILVA FALCÃO & UNIVERSIDADE FEDERAL DO PARÁ - UFPA \\
\hline IRLANDA DO SOCORRO MILÉO & UNIVERSIDADE FEDERAL DO PARA - UFPA \\
\hline IZELDA TODERO & 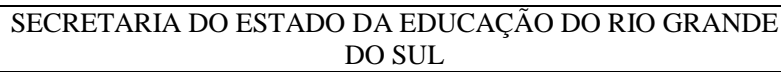 \\
\hline JANETE WEBLER CANCELIER & UNIVERSIDADE FEDERAL DE SANTA MARIA - UFSM \\
\hline JAQUELINE LUZIA DA SILVA & UNIVERSIDADE DO ESTADO DO RIO DE JANEIRO - UERJ \\
\hline JAQUELINE DANIELA BASSO & $\begin{array}{l}\text { UNIVERSIDADE ESTADUAL DE MATO GROSSO DO SUL - } \\
\text { UEMS }\end{array}$ \\
\hline JANINHA GERKE & UNIVERSIDADE FEDERAL DO ESPIRITO SANTO - UFES \\
\hline JEMINA DE ARAÚJO MORAES ANDRADE & INSTITUTO FEDERAL DO AMAPÁ - IFAP \\
\hline JOSÉ SÁVIO BICHO & $\begin{array}{l}\text { UNIVERSIDADE FEDERAL DO SUL E SUDESTE DO PARÁ - } \\
\text { UNIFESSPA }\end{array}$ \\
\hline JOÃO HENRIQUE DA SILVA & UNIVERSIDADE TUIUTI DO PARANÁ \\
\hline JULIANA RIBAS & UNIVERSIDADE FEDERAL DE SANTA MARIA - UFSM \\
\hline JULIANE GOMES DE SOUSA & UNIVERSIDADE FEDERAL DO TOCANTINS - UFT \\
\hline JANINHA GERKE & UNIVERSIDADE FEDERAL DO ESPÍRITO SANTO - UFES \\
\hline JOSÉ CARLOS MIGUEL & UNIVERSIDADE ESTADUAL PAULISTA - UNESP \\
\hline JOSÉ EDIMAR DE SOUZA & UNIVERSIDADE DE CAXIAS DO SUL - UCS \\
\hline JUAN WAHREN & UNIVERSIDAD DE BUENOS AIRES \\
\hline JUCILEY SILVA EVANGELISTA FREIRE & UNIVERSIDADE FEDERAL DO TOCANTINS - UFT \\
\hline JULIANA FERNANDES LANÇA & UNIVERSIDADE ESTADUAL DE MARINGÁ - UEM \\
\hline KAROLYNA MACIEL CORDEIRO & $\begin{array}{l}\text { UNIVERSIDADE ESTADUAL DO NORTE FLUMINENSE } \\
\text { DARCY RIBEIRO }\end{array}$ \\
\hline KAREN CAVALCANTI TAUCEDA & SOCIEDADE BRASILEIRA DE FÍSICA \\
\hline KLIVIA DE CASSIA SILVA NUNES & UNIVERSIDADE FEDERAL DE UBERLANDIA - UFU \\
\hline LEANDRA DOMINGUES SILVÉRIO & $\begin{array}{l}\text { UNIVERSIDADE FEDERAL DO TRIÂNGULO MINEIRO - } \\
\text { UFTM }\end{array}$ \\
\hline LAZARA CRISTINA DA SILVA & UNIVERSIDADE FEDERAL DE UBERLANDIA - UFU \\
\hline LISIANE COSTA CLARO & UNIVERSIDADE FEDERAL DO TOCANTINS - UFT \\
\hline LETÍCIA MENDONÇA LOPES RIBEIRO & UNIVERSIDADE FEDERAL DO ACRE - UFAC \\
\hline LEONARDO GAMA CAMPOS & UNIVERSIDADE FEDERAL FLUMINENSE - UFF \\
\hline LORENA RAQUEL DE ALENCAR DE MORAIS & UNIVERSIDADE ESTADUAL DO PIAUÍ - UESPI \\
\hline LUCAS GUEDES VILAS BOAS & $\begin{array}{l}\text { CENTRO FEDERAL DE EDUCAÇÃO TECNOLÓGICA DE } \\
\text { MINAS GERAIS }\end{array}$ \\
\hline LUCIANE BRESCIANI LOPES & $\begin{array}{l}\text { UNIVERSIDADE FEDERAL DO RIO GRANDE DO SUL - } \\
\text { UFRGS }\end{array}$ \\
\hline
\end{tabular}




\begin{tabular}{|c|c|}
\hline LUISA RODRIGUEZ BELLO & $\begin{array}{l}\text { UNIVERSIDADE PEDAGÓGICA EXPERIMENTAL } \\
\text { LIBERTADOR - VENEZUELA }\end{array}$ \\
\hline LUIS ANTONIO PEREDA JIMÉNEZ & $\begin{array}{l}\text { INSTITUTO TECNOLÓGICO SUPERIOR DE LA SIERRA } \\
\text { NEGRA DE AJALPAN - MÉXICO }\end{array}$ \\
\hline LUIS HENRIQUE DOS SANTOS BARCELLOS & UNIVERSIDADE ESTADUAL PAULISTA - UNESP \\
\hline LUCIENE APARECIDA CASTRAVECHI & INSTITUTO FEDERAL DO PARÁ - IFPA \\
\hline LUCAS ANDRÉ TEIXEIRA & UNIVERSIDADE ESTADUAL PAULISTA - UNESP \\
\hline MACIEL COVER & UNIVERSIDADE FEDERAL DO TOCANTINS - UFT \\
\hline MARCOS IRONDES COELHO & UNIVERSIDADE FEDERAL DO TOCANTINS - UFT \\
\hline MAGNO DA CONCEIÇÃO PENELUC & UNIVERSIDADE FEDERAL DA BAHIA - UFBA \\
\hline MANOLO FREITAS & UNIVERSIDADE FEDERAL DO PARÁ - UFPA \\
\hline MARA PEREIRA DA SILVA & UNIVERSIDADE FEDERAL DO TOCANTINS - UFT \\
\hline MARCELO FRANCO LEÃO & INSTITUTO FEDERAL DE MATO GROSSO - IFMT \\
\hline MARCIO GOMES DA SILVA & UNIVERSIDADE FEDERAL FLUMINENSE - UFF \\
\hline MARCELO CAVALCANTI VIANNA & UNIVERSIDADE FEDERAL FLUMINENSE - UFF \\
\hline MARCUS FACCHIN BONILLA & UNIVERSIDADE FEDERAL DO TOCANTINS - UFT \\
\hline MARÍA MERCEDES PALUMBO & UNIVERSIDADE DE BUENOS AIRES \\
\hline MARIA ZENAIDE ALVES & UNIVERSIDADE FEDERAL DE GOIÁS - UFG \\
\hline MARIANE DA SILVA PISANI & UNIVERSIDADE FEDERAL DO TOCANTINS - UFT \\
\hline MARIELA CRISTINA AYRES DE OLIVEIRA & UNIVERSIDADE FEDERAL DO TOCANTINS - UFT \\
\hline MARINALVA NUNES FERNANDES & UNIVERSIDADE DO ESTADO DA BAHIA - UNEB \\
\hline MÁRIO BORGES NETTO & UNIVERSIDADE FEDERAL DE UBERLANDIA - UFU \\
\hline MARÍA JULIA AGUILAR AGUILERA & UNIVERSIDAD DE ARTEMISA, CUBA \\
\hline MARITZA COSTA MORAES & UNIVERSIDADE FEDERAL DO PAMPA - UNIPAMPA \\
\hline MARTIN KUHN & $\begin{array}{l}\text { UNIVERSIDADE REGIONAL INTEGRADA DO ALTO } \\
\text { URUGUAI E DAS MISSÕES - URI }\end{array}$ \\
\hline MURILO OLIVEIRA SOUZA & UNIVERSIDADE ESTADUAL DE GOIÁS - UEG \\
\hline NATHÁLIA LOPES CALDEIRA BRANT & INSTITUTO FEDERAL DE MINAS GERAIS - IFMG \\
\hline NEY CRISTINA MONTEIRO DE OLIVEIRA & UNIVERSIDADE FEDERAL DO PARÁ - UFPA \\
\hline OSMAR LOTTERMANN & $\begin{array}{l}\text { INSTITUTO FEDERAL DE EDUCAÇÃO, CIÊNCIA E } \\
\text { TECNOLOGIA DO RIO GRANDE DO SUL }\end{array}$ \\
\hline OSNI OLIVEIRA NOBERTO DA SILVA & UNIVERSIDADE DO ESTADO DA BAHIA - UNEB \\
\hline PAULA LEONARDI & UNIVERSIDADE DO ESTADO DO RIO DE JANEIRO - UERJ \\
\hline PEDRO RIBEIRO MUCHARREIRA & UNIVERSIDADE DE LISBOA \\
\hline PEDRO HENRIQUE GOMES XAVIER & UNIVERSIDADE DE BRASILIA - UNB \\
\hline RAMOFLY BICALHO SANTOS & $\begin{array}{l}\text { UNIVERSIDADE FEDERAL RURAL DO RIO DE JANEIRO - } \\
\text { UFRRJ }\end{array}$ \\
\hline RAIMUNDO NONATO DE PÁDUA CÂNCIO & UNIVERSIDADE FEDERAL DO TOCANTINS - UFT \\
\hline REJANE CLEIDE MEDEIROS DE ALMEIDA & UNIVERSIDADE FEDERAL DO TOCANTINS - UFT \\
\hline RIBAMAR RIBEIRO JÚNIOR & INSTITUTO FEDERAL DO PARÁ - IFPA \\
\hline SÉRGIO BOTTON BARCELLOS & UNIVERSIDADE DO RIO GRANDE - FURG \\
\hline SIMARA MARIA TAVARES NUNES & UNIVERSIDADE FEDERAL DE GOIÁS - UFG \\
\hline OSMAR LOTTERMANN & INSTITUTO FEDERAL DE FARROUPINHA - IFFARROUPILHA \\
\hline PAULO GERSON STEFANELLO & UNIVERSIDADE DO ESTADO DE MATO GROSSO - UEMS \\
\hline CLAUDEMIR DE QUADROS & UNIVERSIDADE FEDERAL DE SANTA MARIA - UFSM \\
\hline REJANE CLEIDE MEDEIROS DE ALMEIDA & UNIVERSIDADE FEDERAL DO TOCANTINS - UFT \\
\hline RICARDO FRANKLIN DE FREITAS MUSSI & UNIVERSIDADE DO ESTADO DA BAHIA - UNEB \\
\hline ROBERTO FRANCISCO DE CARVALHO & UNIVERSIDADE FEDERAL DO TOCANTINS - UFT \\
\hline ROSILENE LAGARES & UNIVERSIDADE FEDERAL DO TOCANTINS - UFT \\
\hline SAMUEL FRANCISCO HUF & $\begin{array}{l}\text { UNIVERSIDADE ESTADUAL DO CENTRO OESTE - } \\
\text { UNICENTRO }\end{array}$ \\
\hline SAMILO TAKARA & UNIVERSIDADE FEDERAL DE RONDONIA - UNIR \\
\hline STENIO DE BRITO FERNANDES & $\begin{array}{l}\text { UNIVERSIDADE DO ESTADO DO RIO GRANDE DO NORTE - } \\
\text { UERN }\end{array}$ \\
\hline SANDRA MARA MEZALIRA & $\begin{array}{l}\text { UNIVERSIDADE FEDERAL DO RIO GRANDE DO SUL - } \\
\text { UFRGS }\end{array}$ \\
\hline SINARA MUNCHEN & UNIVERSIDADE FEDERAL DA FRONTEIRA SUL - UFFS \\
\hline TANIA REGINA BRAGA TORREAO & $\begin{array}{l}\text { UNIVERSIDADE ESTADUAL DO SUDOESTE DA BAHIA - } \\
\text { UESB }\end{array}$ \\
\hline THIAGO COSTA FERREIRA & PESQUISADOR INDEPENDENTE \\
\hline UMBERTO EUZEBIO & UNIVERSIDADE DE BRASILIA - UNB \\
\hline VAGNER LUIZ KOMINKIÉWICZ & UNIVERSIDADE FEDERAL DE SANTA CATARINA - UFSC \\
\hline VALDOMIRO PINHEIRO TEIXEIRA JUNIOR & PESQUISADOR INDEPENDENTE \\
\hline VINÍCIUS CATÃO & UNIVERSIDADE FEDERAL DE VIÇOSA - UFV \\
\hline WEIMAR SILVA CASTILHO & $\begin{array}{l}\text { INSTITUTO FEDERAL DE EDUCAÇÃO, CIÊNCIA E } \\
\text { TECNOLOGIA DO TOCANTINS - IFTO }\end{array}$ \\
\hline WILSON JOSÉ SOARES & $\begin{array}{l}\text { INSTITUTO FEDERAL DE EDUCAÇÃO, CIÊNCIA E } \\
\text { TECNOLOGIA DE MATO GROSSO - IFMT } \\
\end{array}$ \\
\hline
\end{tabular}


Agradecemos as contribuições dos pareceristas e avaliadores ad hoc da Revista Brasileira de Educação do Campo - RBEC no ano de 2020.

We appreciate the contributions of the reviewers and referees of the Brazilian Journal of Rural Education in the year 2020.

* Não houve conflito de interesses nas avaliações dos manuscritos.

** Conflict of Interest: None reported.

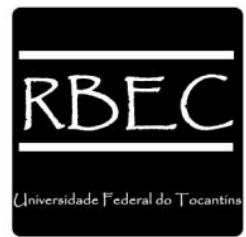

Revista Brasileira de Educação do Campo

Brazilian Journal of Rural Education 